\title{
Genotipificación del virus papiloma humano en mujeres con adenocarcinoma cervical de la Región de La Araucanía-Chile
}

\author{
Angélica Melo A., Patricia García M., Italo Capurro V., Pablo Guzmán G., \\ Priscila Brebi M., Carmen Ili G., Jaime López M. y Juan C. Roa S.
}

\section{Human papillomavirus genotyping in cervical adenocarcinoma in the Region of La Araucanía-Chile}

Human papillomavirus (HPV) is the main cause of cervical cancer. Thus, HPV detection and typing becomes important in order to know the frequency of genotypes present in the region. In this paper we studied 44 biopsies of cervical adenocarcinoma. For HPV detection nested polymerase chain reaction (PCR) was used to amplify the L1 gene. For viral typing restriction enzymes (Rsa I, Dde I, Pst I) and DNA sequencing were used. Viral DNA was detected by nested L1 PCR in $100 \%$ of biopsies; $38 / 44$ cases could be typed: $81.6 \%$ HPV $16 ; 13.2 \%$ HPV 18 ; $2.6 \%$ VPH 33 and 2.6\% HPV 18/33. Conclusions: The technique was successful in identifying the virus type in $86 \%$ of biopsies. There was a strong association ACC-HPV, especially with the viral type 16, detected in $81.6 \%$ of established cases.

Key words: HPV, cervical cancer, cervical adenocarcinoma, PCR, RFLP, Sequence.

Palabras clave: VPH, cáncer de cuello uterino, adenocarcinoma, RPC, RFLP, Secuencia.

\section{Introducción}

$\mathrm{E}$ n Chile, el cáncer cervico-uterino (CCU) ocupa el cuarto lugar dentro de las neoplasias malignas, con tasas de mortalidad de 8,7 por $100.000 \mathrm{mu}-$ jeres mayores de 15 años ${ }^{1}$. En la Región de la Araucanía constituye la tercera causa de muerte por cáncer, con una tasa de mortalidad de $9,4 \times 100.000^{2}$. La mayoría de los CCU son del tipo escamocelular o epidermoide $(80 \%)$, mientras que el resto corresponde principalmente al adenocarcinoma cervical (ACC). Desde los años 70, la frecuencia del ACC ha ido en aumento, lo que está en directa relación con la mayor frecuencia de infecciones genitales por el VPH, sumado a otros factores como son el número de parejas sexuales y tiempo de uso de anticonceptivos orales, entre otros $^{3,4}$.

El VPH se ha establecido, biológica y epidemiológicamente, como el principal agente causal del CCU. A la fecha, alrededor de 18 tipos virales se consideran con potencial oncogénico y se les clasifica como tipos de alto riesgo por su presencia en lesiones de alto grado y en carcinomas ${ }^{5,6}$. El VPH 16 ha sido el genotipo más frecuentemente detectado a nivel mundial; sin embargo, en países del Asia-Pacífico, el VPH 18 se ha observado en un porcentaje mayor que el VPH 16 en mujeres con $\mathrm{ACC}^{4,7,8}$.
Pese a la prevalencia del CCU en Chile, son escasos los estudios regionales referentes a la distribución de los tipos virales y su asociación con $\mathrm{CCU}^{9-12}$. Nuestro objetivo fue detectar y tipificar el VPH en biopsias de mujeres con ACC, utilizando las metodologías combinadas de RPC y enzimas de restricción (PCR-RFLP) y RPC-secuenciación.

\section{Material y Método}

Muestras: Se realizó un estudio retrospectivo analizando 44 biopsias de cuello uterino con diagnóstico histopatológico de adenocarcinoma, utilizando el material que se encontraba disponible en el momento del estudio. Todos los casos fueron obtenidos desde la Unidad de Anatomía Patológica-Citología entre los años 2000 y 2004 en el Policlínico de Patología Cervical del Hospital Hernán Henríquez Aravena. El estudio fue aprobado por el Comité de Ética del Servicio de Salud Araucanía Sur.

Extracción de ADN: Manualmente, se microdisecaron 8 cortes de 7 micras de cada caso, de acuerdo al área señalada en la placa teñida con hematoxilina-eosina. El tejido fue digerido en tampón de lisis con proteinasa K (1 $\mathrm{mg} / \mathrm{ml}$ ), se precipitó el ADN con isopropanol y finalmente
Universidad de La Frontera Facultad de Medicina. Departamento de Anatomía Patológica Laboratorio de Patología (AMA, PGM, JCR).

Molecular Scientific and Technological Bioresource Nucleus (BIOREN), Temuco, Chile (AMA, PGM, JCR).

Programa Doctorado en Biología Molecular y Celular Aplicada (PBM, CIG, JLM).

Hospital Hernán Henríquez Aravena, Temuco, Chile. Unidad de Obstetricia y Ginecología (ICV). Unidad de Anatomía Patológica-Citología (PGG).

Financiado parcialmente por el Proyecto DIUFRO-07003 de la Universidad de La Frontera y Proyecto CORFO INNOVA-CHILE $n^{\circ}$ 07CN13PBT-222

Recibido: 5 de marzo de 2009 Aceptado: 28 de abril de 2010

Correspondencia a: Juan Carlos Roa S jcroa@ufro.cl 
fue hidratado y guardado a $-20^{\circ} \mathrm{C}$ hasta su análisis. Para evaluar la calidad del $\mathrm{ADN}$ todas las muestras fueron sometidas a una RPC simple para el gen de la $\beta$-globina ${ }^{13}$.

Detección VPH. Para la detección se utilizó una RPC anidada, con partidores de consenso dirigidos a una secuencia altamente conservada del gen viral L1 (RPCL1). Los partidores externos (MY11/ MY09) amplifican un fragmento de 450pb ("outer") y los internos (GP5+/ GP6+), un fragmento de 150pb ("inner"). Esta RPCL1 anidada permite detectar tipos virales de alto riesgo (AR) y bajo riesgo (BR), como asimismo subtipos virales aún no caracterizados ${ }^{14}$. Como controles positivos se utilizaron plásmidos recombinantes para los tipos VPH 6, 11, 16, 18, 31, 35, 33 у 52 (ATTC USA).

Tipificación de VPH. Para la tipificación de los tipos virales se utilizaron dos estrategias. En la metodología A se utilizaron los partidores externos para amplificar el fragmento de 450pb, seguido de RFLP. Se confeccionaron patrones de restricción para los tipos virales más frecuentes de BR y AR ( HPV6, 11, 16, 18, 31, 33, 34, $35,39,45,51,52,56,58,59,66,68,70,73$ y 82$)$, a partir del análisis de las secuencias publicadas en GeneBank

Tabla 1. Tipificación del VPH en muestras de adenocarcinoma según las metodologías empleadas

\begin{tabular}{|lccccc} 
& \multicolumn{5}{c}{ Tipificación de VPH (\%) } \\
Muestras & $\mathbf{n}$ & VPH 16 & VPH 18 & VPH 33 & IM* $^{*}$ \\
\hline Tipificables con MetA & 25 & 22 & 1 & 1 & 1 \\
\hline Tipificables con MetB & 13 & 9 & 4 & 0 & $\mathrm{NA}^{*}$ \\
Total & 38 & $31 / 38(81,6)$ & $5 / 38(13,2)$ & $1 / 38(2,6)$ & $1 / 38(2,6)$
\end{tabular}

MetA: metodología PCRL1"outer" más RFLP. MetB: metodología PCRL1 anidada más secuencia fragmento "inner". IM: infección múltiple. *NA: no aplica.

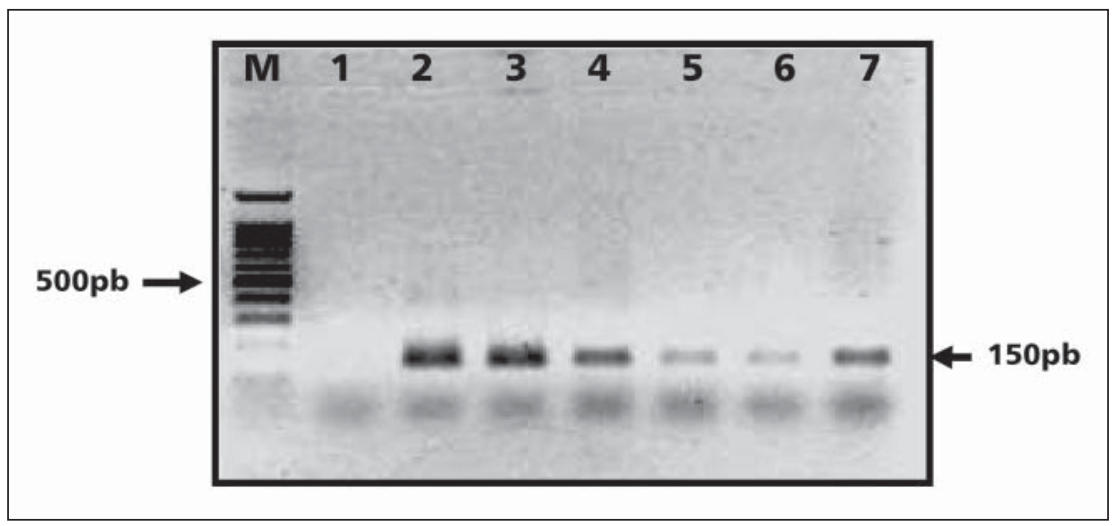

Figura 1. RPC L1 anidada: Carril 1 Blanco. Carril 2 Control Positivo plásmido VPH 16, Carriles 3-7 muestras ACC, VPH positivas. Gel de agarosa al 2\% teñido con bromuro de etidio. (www.ncbi.nlm.nih.gov/taxonomy). Aquellas muestras que presentaron una banda visible y clara del fragmento de $450 \mathrm{pb}$, fueron sometidas a digestión $(10 \mu \mathrm{l}$ del producto RPC) con las endonucleasas Rsa I, Dde I y Pst I (Promega USA) que producen un patrón de digestión, exclusivo para cada tipo viral. Las condiciones de RPC (RPCL1 “outer") fueron 35 ciclos con temperatura de hibridación de $40^{\circ} \mathrm{C}$. Los productos RPC se visualizaron en geles de agarosa al $2 \%$ y los fragmentos de la digestión enzimática en geles de poliacrilamida al $10 \%$, ambos teñidos con bromuro de etidio.

Aquellas muestras que no pudieron ser tipificadas mediante RPC-RFLP se analizaron aplicando la metodología $\mathrm{B}$, la cual combinó la RPCL1 anidada y secuenciación del fragmento de $150 \mathrm{pb}$. Las condiciones de RPC (RPCL1 anidada) fueron 30 y 40 ciclos con temperatura de hibridación de 40 y $45^{\circ} \mathrm{C}$ (para reacción outer e inner, respectivamente). Los productos RPC se visualizaron en geles de agarosa al 2\% teñido con bromuro de etidio, se purificaron con E.Z.N.A. Gel Extraction Kit (Omega Bio-tek) y se enviaron a secuenciar (ambas hebras) a Macrogen- Korea. Los resultados fueron analizados con el programa Geneious, que permite comparar las secuencias en estudio con secuencias virales publicadas en Genebank.

\section{Resultados}

El grupo de mujeres tuvo un rango de edad entre 24 y 78 años. Con respecto al grado de diferenciación de los ACC, 21 casos eran bien diferenciado, 16 tenían diferenciación moderada y 7 correspondieron a tumores poco diferenciados.

Detección de VPH. En 44 muestras se obtuvo amplificación del gen de la $\beta$-globina y todas ellas amplificaron con la RPCL1 anidada, indicando que $100 \%$ de las muestras fueron positivas para el VPH (Figura 1).

Tipificación de VPH. Los resultados se pueden observar en la Tabla 1 y en las Figuras 1, 2 y 3. Empleando la metodología A (RPCL1 fragmento "outer" (450 bp) y RFLP), el VPH pudo ser tipificado en un total de 25/44 casos: 22/25 VPH 16; 1/25 VPH 18; 1/25 VPH 33 y un caso con doble infección (VPH 18/33) (Figura 2). Con la metodología B (RPC L1 anidada y secuenciación del producto de RPC de $150 \mathrm{pb}$ ) el tipo viral fue identificado en 13/19 casos: 9/13 VPH 16 y 4/13 VPH 18. En 4 muestras el resultado de la secuencia no permitió determinar el tipo viral (muestras no tipificables) y 2 muestras no fueron secuenciadas por no obtenerse la cantidad mínima de producto RPC requerido para secuenciación (Figura 3).

En total, combinando ambas metodologías, se tipificaron 38 casos, siendo VPH 16 el genotipo más frecuente 
dentro de las muestras tipificadas $(81,5 \%)$, seguido del VPH 18 con 13,1\% (en infecciones únicas). De los 5 casos VPH 18 encontrados, 4 correspondieron a mujeres menores de 50 años.

\section{Discusión}

La asociación ACC-VPH fue evidenciada en la totalidad de los casos evaluados, lográndose tipificar $86 \%$ (38/44) de ellos, utilizando una estrategia metodológica que combinó el uso de dos técnicas basadas en RPC. Al respecto, la aplicación de la metodología A permitió tipificar 25/44 muestras. La principal limitante de este método está en relación a la calidad del material de archivo utilizado, con tiempos de fijación en formalina no estandarizados. La literatura señala que la fijación y el tiempo de almacenamiento de las muestras pueden degradar o fragmentar el ADN, lo cual interfiere con la amplificación de fragmentos mayores a $250 \mathrm{pb}$, disminuyendo la eficiencia de la RPC ${ }^{15-17}$. Para analizar muestras de estas características, es recomendable el uso de una RPC anidada que aumenta la sensibilidad de amplificación. Para detectar VPH, Zehbe y cols ${ }^{18}$, señalan que el uso combinado de los iniciadores MY09/MY11 más los iniciadores GP5+/GP6+, en una RPC anidada, aumenta en 100 veces la sensibilidad de una RPC simple. Esto queda de manifiesto con los resultados obtenidos, puesto que mediante la RPC anidada fue amplificado el ADN viral en todas las muestras positivas para $\beta$-globina. Sin embargo, cuando se requirió amplificar solamente el fragmento de 450pb (RPCL1), en 19 muestras, la cantidad de producto de RPC generado fue insuficiente para visualizar bandas post-digestión enzimática. Estos casos fueron analizados mediante la metodología B, la cual se basó en la secuenciación del producto de la RPC anidada, un fragmento más pequeño (150 pb) y visiblemente más abundante que el fragmento de $450 \mathrm{pb}$, siendo insuficiente para secuenciación ( $<10 \mathrm{ng}$ de producto purificado) en sólo 2 muestras. El análisis de las secuencias fue exitoso para 13 casos, pero no permitió identificar el genotipo viral de 4 muestras, ya que ellas mostraron varios patrones de secuenciación que podrían corresponder a infecciones múltiples. Este último hecho limita el uso de esta técnica, ya que permite tipificar muestras que presenten un solo tipo viral. Pese a las limitantes inherentes a ambas técnicas (RPC-RFLP y RPC-secuencia), la combinación de ellas permitió tipificar $86 \%$ de los casos estudiados.

Con respecto a los genotipos virales identificados en los ACC, predominó ampliamente VPH 16 (81\%), presentándose en un porcentaje significativamente menor VPH 18 (13\%). Estos resultados concuerdan con reportes previos, donde los tipos virales más comunes en la gran mayoría de los ACC son VPH 16 y VPH 18,8.

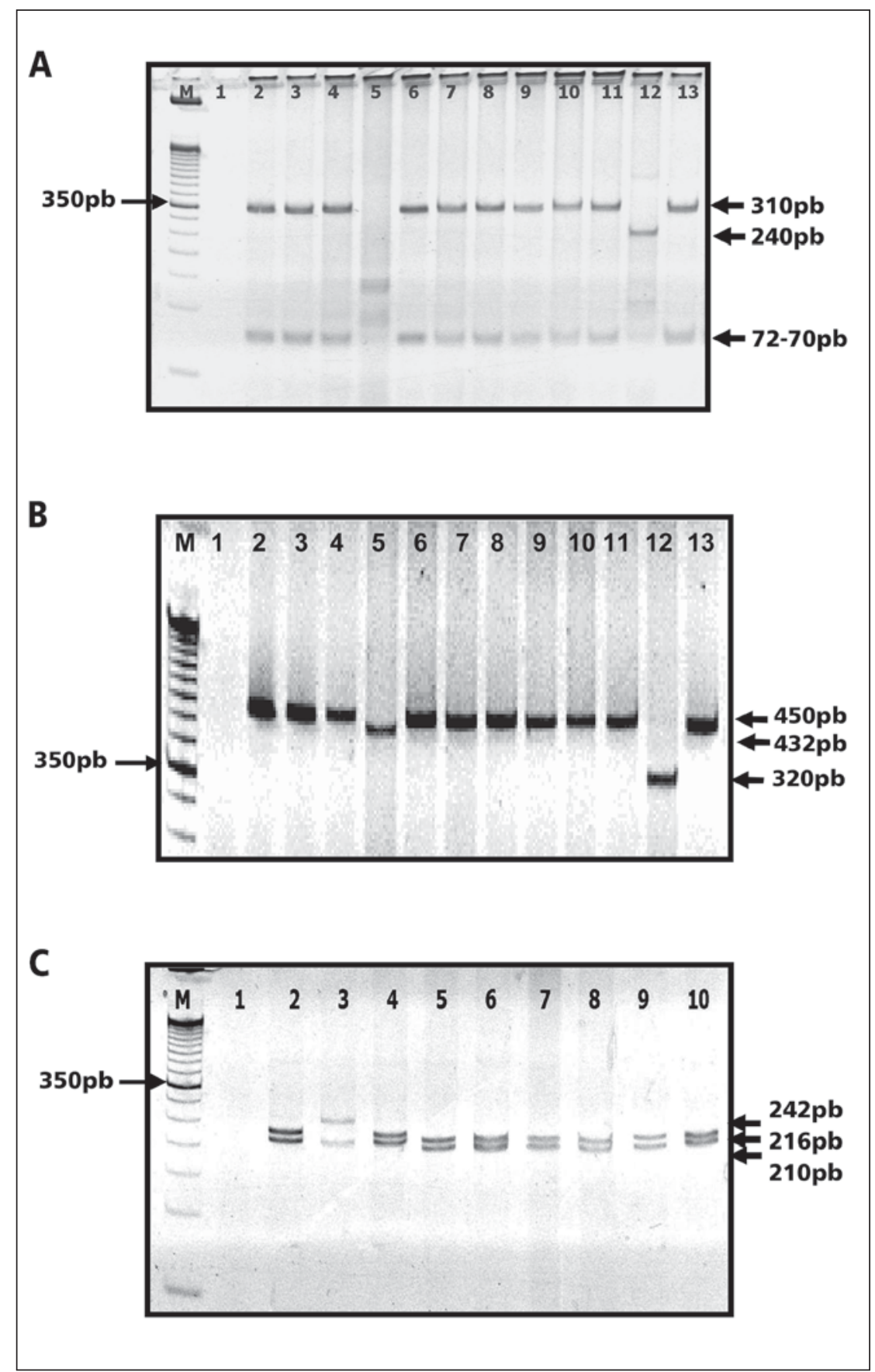

Figura 2. A: RPC-RFLP con la enzima de restricción Rsa I: Marcador 50pb. Carril 1 Blanco. Carril 2 control plásmido VPH 16 (310-72-70pb). Carril 3, 4, 6-11 y 13: muestras de ACC con patrón de corte VPH 16 o VPH 56. Carril 5 Muestra ACC patrón de digestión VPH 18 (135-125 y 85 pb). Carril 12 Muestra de ACC patrón de digestión VPH 33 (241-100bp). Gel de poliacrilamida 12\% teñido con bromuro de etidio. B: PCR-RFLP con la enzima de restricción Dde I: Marcador 50pb. Carril 1 Blanco. Carril 2 control plásmido VPH 16 (450pb). Carril 3,4, 6-11 y 13 Muestras de ACC, no hay digestión enzimática, patrón de VPH 16. Carril 5 patrón de digestión VPH 18 (432pb). Carril 12 patrón de digestión VPH 33 (320 pb). Gel de poliacrilamida 12\% teñido con bromuro de etidio. C: PCR-RFLP con la enzima de restricción Pst I: Marcador 50pb. Carril 1 Blanco. Carril 2 control plásmido VPH 16 (216-210pb). Carril 3 Control positivo VPH 18 (242/ 210 pb). Carril 4-10 muestras de ACC correspondiente a patrón de digestión VPH 16. 
Figura 3. RPCL1 anidadasecuenciación. Se muestra el cromatograma de una secuencia representativa.

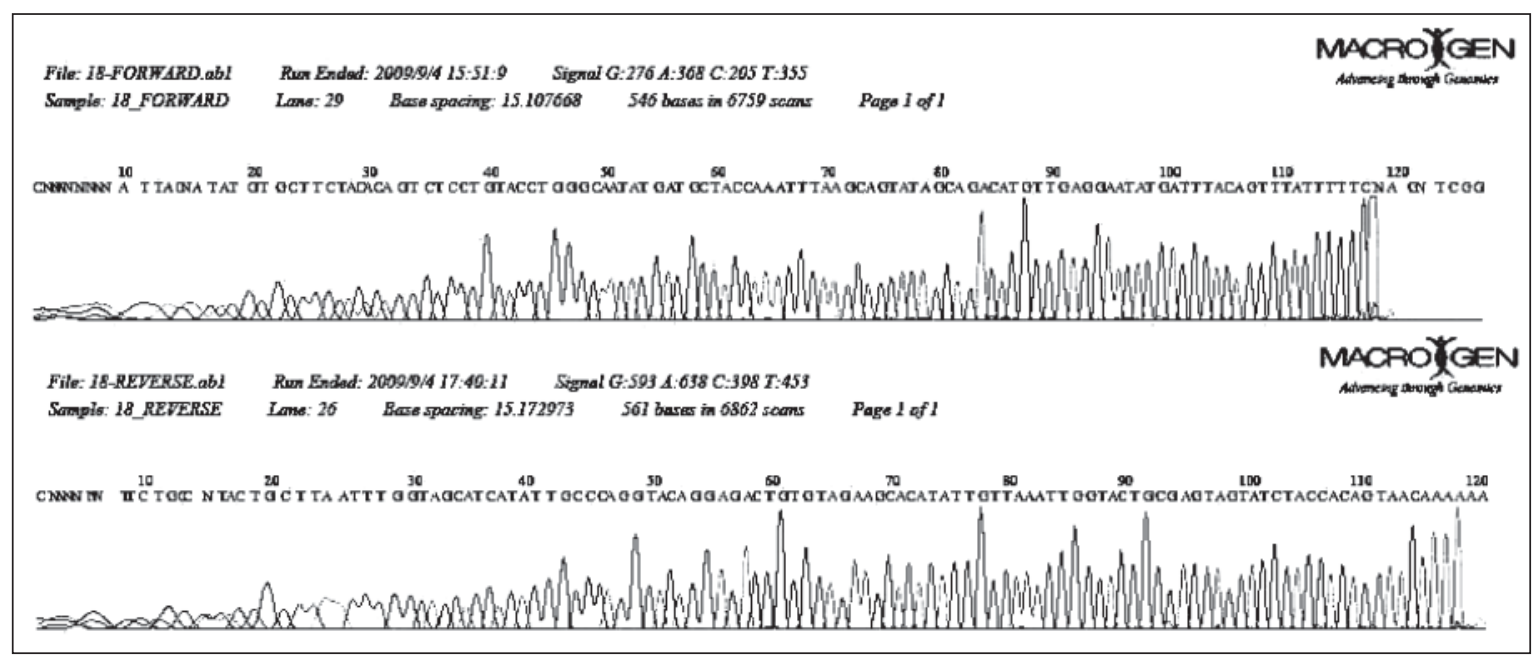

Sin embargo, la revisión de la literatura disponible indica que existe variación en la frecuencia de estos tipos dependiendo de las regiones geográficas, hecho que también ha sido observado en la asociación entre VPH y carcinoma escamoso $^{19,20}$. Al respecto, Pirog y cols, obtuvieron en mujeres con ACC de Nueva York frecuencias de 50 y $40 \%$ para VPH 16 y 18, respectivamente ${ }^{8}$. Por otra parte, en la población finlandesa se ha reportado una menor frecuencia de VPH 16 (17\%) versus VPH $18(56 \%)^{21}$. Similarmente, el grupo de Andersson y cols, detectó un mayor porcentaje de VPH 18 (52\%) seguido del VPH 16 (35\%), en biopsias de ACC de mujeres suecas, siendo la presencia de VPH significativamente más frecuente en mujeres menores de 40 años ${ }^{22}$. También en mujeres japonesas existe una mayor asociación entre adenocarcinoma y VPH 18, aunque las diferencias con respecto al VPH 16 no parecen ser significativas ${ }^{23}$.

Las variaciones geográficas juegan un papel importante en el predominio de uno u otro tipo de $\mathrm{VPH}$, con ejemplos representativos como son la alta frecuencia de VPH 16 en casos de ACC en la India ${ }^{24}$ o de VPH 18 en Finlandia ${ }^{22}$. En Chile y en la Región de La Araucanía, estudios realizados en muestras de adenocarcinoma, VPH 16 fue altamente predominante por sobre los otros tipos virales, existiendo una baja frecuencia de VHP $18^{25,26}$. Resultados similares se han observado en otras series de casos latinoamericanos donde la frecuencia de VPH 16 fue de 82,6\% y $13 \%$ para VPH $18^{27}$. Roa y cols, en un estudio multicéntrico encontraron $22 \%$ de VPH 18 y Brebi y cols, observaron una frecuencia de 19,5\%, siendo las series estudiadas de 35 y 29 casos respectivamente. En estos estudios, el porcentaje de VPH 18 se determinó considerando infecciones únicas y múltiples. En el presente trabajo la frecuencia de VPH 18 sería de $16 \%$ si se considera el caso de infección múltiple. Estas diferencias obtenidas se pueden explicar por las metodologías utilizadas. Por un lado, la metodología RPC-RLB (Reverse Line Blot) es más sensible para detectar infecciones múltiples y éstas contribuyen a elevar el número de casos si son incluidas al calcular la frecuencia. De esta manera, en el trabajo de Brebi y cols, podemos observar que la frecuencia de VPH 18 en infecciones únicas sería 13,8\%, siendo similar a lo reportado en el presente estudio ${ }^{25,26}$.

Así, los resultados publicados y los obtenidos en esta investigación, reafirman que en la región de La Araucanía, VPH 16 predomina ampliamente sobre el VPH 18.

Conocer la distribución y frecuencia de los diferentes tipos virales del VPH en adenocarcinoma cervical, contribuirán en el futuro a diseñar y aplicar mejores programas de tamizaje y vacunación en poblaciones de alto riesgo.

\section{Resumen}

El virus papiloma humano (VPH) es el principal factor causal del cáncer cervicouterino (CCU). Así, detectar y genotipificar el VPH es importante para conocer la frecuencia de los genotipos presentes en la región. En este trabajo se estudiaron 44 biopsias de adenocarcinoma cervical (ACC). Para la detección del VPH se empleó una reacción de polimerasa en cadena anidada dirigida al gen L1 (RPCL1), para la genotipificación viral se utilizaron enzimas de restricción (Rsa I, Dde I, Pst I) y secuenciación. Se detectó ADN viral mediante RPCL1 anidada en $100 \%$ de las biopias. Se logró tipificar 38/44 casos: 81,6\% VPH 16; 13,2\% VPH 18; 2,6\% VPH 33 y $2,6 \%$ VPH 18/33. Conclusiones: La metodología fue exitosa para identificar el tipo viral en $86 \%$ de las biopsias. Se observó una estrecha asociación ACC-VPH, especialmente con el tipo viral 16, detectado en $81,6 \%$ de los casos tipificados. 


\section{Referencias}

1.- Suárez E, Prieto M, Rojas I, Fernández B, Prado R, Olfos P. Programa Nacional de Cáncer Cervicouterino. Rev Chil Obstet Ginecol 2001; 66 (6): 480-91.

2.- Capurro I R J, Pino T, Vásquez C, Garay J, Venegas M. Programa de Detección y Control de Cáncer de Cuello Uterino en Servicio de Salud Araucanía Sur. Rev Chil Obstet Ginecol 2002; 67 (2): 114-20.

3.- Cancer ICoESoC. Comparison of risk factors for invasive squamous cell carcinoma and adenocarcinoma of the cervix: Collaborative reanalysis of individual data on 8,097 women with squamous cell carcinoma and 1,374 women with adenocarcinoma from 12 epidemiological studies. Int J Cancer 2007; 120: 885-91.

4.- Castellsague X, Díaz M, de Sanjose S, Muñoz N, Herrero R, Franceschi S, et al. Worldwide human papillomavirus etiology of cervical adenocarcinoma and its cofactors: implications for screening and prevention. J Natl Cancer Inst 2006; 98 (5): 303-15.

5.- Muñoz N, Castellsague X, de González A B, Gissmann L. Chapter 1: HPV in the etiology of human cancer. Vaccine 2006; 24 Suppl 3: S3/ $1-10$.

6.- Muñoz N, Bosch F X, de Sanjose S, Herrero R, Castellsague X, Shah K V, et al. Epidemiologic classification of human papillomavirus types associated with cervical cancer. N Engl J Med 2003; 348 (6): 518-27.

7.- Huang L W, Chao S L, Chen P H, Chou H P. Multiple HPV genotypes in cervical carcinomas: improved DNA detection and typing in archival tissues. J Clin Virol 2004; 29 (4): 271-6.

8.- Pirog E C, Kleter B, Olgac S, Bobkiewicz P, Lindeman J, Quint W G, et al. Prevalence of human papillomavirus DNA in different histological subtypes of cervical adenocarcinoma. Am J Pathol 2000; 157 (4): 1055-62.

9.- Valdivia I M, Aguayo G F, Pruyas A M, Snijders P J F, Corvalán A, Ferreccio C. Genotipos de virus papiloma humano (VPH) en pacientes con cáncer cérvico-uterino en un hospital público y una clínica privada de
Santiago, Chile. Rev Chil Infectol 2010; 27 (1): 11-6.

10.- Melo A, Montenegro S, Hooper T, Capurro I, Roa J C, Roa I. Human papilloma virus (HPV) typing in preneoplastic and neoplastic lesions of the uterine cervix in the IX regionChile. Rev Méd Chile 2003; 131 (12): 1382-90.

11.- Aedo S M A, García P, Guzmán P, Capurro I, Roa J C. Detección y tipificación de virus papiloma humano en lesiones preneoplásicas del cuello uterino mediante PCR-RFLP. Rev Méd Chile 2007; 135 (2): 167-73.

12.- Socias M G J, Figueroa J, Fernández F. Perfil epidemiológico y clínico de la tipificación del HPV capture híbrida. Método Digene. Rev Chil Obstet Ginecol 1997; 62: 167-73.

13.- Saiki R K, Scharf S, Faloona F, Mullis K B, Horn G T, Erlich H A, et al. Enzymatic amplification of beta-globin genomic sequences and restriction site analysis for diagnosis of sickle cell anemia. Science 1985; 230 (4732): 1350-4.

14.- de Roda Husman A M, Walboomers J M, van den Brule A J, Meijer C J, Snijders P J. The use of general primers GP5 and GP6 elongated at their 3' ends with adjacent highly conserved sequences improves human papillomavirus detection by PCR. J Gen Virol 1995; 76 (Pt 4): 1057-62.

15.- Zafra G F O, González C. Influencia del método de desparafinación y el tiempo de almacenamiento en la extracción de DNA a partir de tejidos de archivo. Salud UIS 2004; 36: 73-9.

16.- Greer C E, Peterson S L, Kiviat N B, Manos M M. PCR amplification from paraffin-embedded tissues. Effects of fixative and fixation time. Am J Clin Pathol 1991; 95 (2): 117-24.

17.- García P, Benavente F, Melo A, Roa I, Roa J C. Efecto de la fijación en la calidad del ADN: Estudio controlado con 5 fijadores. Res Esp Patol 2006; 39 (3): 175-9.

18.- Zehbe I, Wilander E. Two consensus primer systems and nested polymerase chain reaction for human papillomavirus detection in cervical biopsies: A study of sensitivity. Hum Pathol 1996; 27 (8): 812-5.

19.- Ihekweazu C. Worldwide distribution of HPV types in women with normal cervical cytology and in women with cervical adenocarcinoma. Euro Surveill 2006; 11 (3): E060323 6.

20.- Bosch F X, Manos M M, Muñoz N, Sherman M, Jansen A M, Peto J, et al. Prevalence of human papillomavirus in cervical cancer: a worldwide perspective. International biological study on cervical cancer (IBSCC) Study Group. J Natl Cancer Inst 1995; 87 (11): 796-802.

21.- Iwasawa A, Nieminen P, Lehtinen M, Paavonen J. Human papillomavirus DNA in uterine cervix squamous cell carcinoma and adenocarcinoma detected by polymerase chain reaction. Cancer 1996; 77 (11): 2275-9.

22.- Andersson S, Rylander E, Larson B, Sigurdardottir S, Backlund I, Sallstrom J, et al. Types of human papillomavirus revealed in cervical adenocarcinomas after DNA sequencing. Oncol Rep 2003; 10 (1): 175-9.

23.- Sasagawa T, Basha W, Yamazaki H, Inoue M. High-risk and multiple human papillomavirus infections associated with cervical abnormalities in Japanese women. Cancer Epidemiol Biomarkers Prev 2001; 10 (1): 45-52.

24.- Das B C, Gopalkrishna V, Das D K, Sharma J K, Singh V, Luthra U K. Human papillomavirus DNA sequences in adenocarcinoma of the uterine cervix in Indian women. Cancer 1993; 72 (1): 147-53.

25.- Roa J C G P, Gómez J, Fernández W, Gaete F, Espinoza A, Lepetic A, et al. HPV genotyping from invasive cervical cancer in Chile. Int J Gynaecol Obstet 2009; 105 (2): 150-3.

26.- Brebi P, Ili C G, López J, García P, Melo A, Montenegro S, et al. Detección y tipificación de virus papiloma humano en adenocarcinoma de cuello uterino mediante reverse line blot, Región de La Araucanía, Chile. Rev Méd Chile 2009; 137 (3): 377-82.

27.- Santos C, Muñoz N, Klug S, Almonte M, Guerrero I, Álvarez M, et al. HPV types and cofactors causing cervical cancer in Peru. Br J Cancer 2001; 85 (7): 966-71. 